\title{
Tromboembolismo venoso e câncer colorretal: um desafio para o cirurgião. Relato de caso e revisão de literatura
}

\author{
Venous thromboembolism and colorectal neoplasm: a challenge for the surgeon. \\ Case report and literature review
}

\author{
SINARA MÔNICA DE OLIVEIRA LEITE ${ }^{1}$, CLEBER LUÍZ SCHEIDEGGER MAIA JÚNIOR², ALEXANDRE \\ MIRANDA SILVEIRA ${ }^{3}$
}

\begin{abstract}
${ }^{1}$ Coloproctologista do Hospital Governador Israel Pinheiro da Santa Casa de Belo Horizonte e do Hospital Biocor - Belo Horizonte (MG), Brasil; Professora de Coloproctologia da Faculdade de Ciências Médicas de Minas Gerais (FCMMG)

- Belo Horizonte (MG), Brasil; Titular da Sociedade Brasileira de Coloproctologia. ${ }^{2}$ Médico Residente do $2^{\circ}$ ano de

Coloproctologia do Hospital Governador Israel Pinheiro - Instituto de Previdência dos Servidores do Estado de Minas

Gerais (IPSEMG) - Belo Horizonte (MG), Brasil. ${ }^{3}$ Cirurgião geral e Coloproctologista pelo Hospital Governador Israel Pinheiro - IPSEMG - Belo Horizonte (MG), Brasil.
\end{abstract}

LEITE SMO, JÚNIOR CLSM, SILVEIRAAM. Tromboembolismo venoso e câncer colorretal: um desafio para o cirurgião. Relato de caso e revisão de literatura. Rev bras Coloproct, 2011;31(2): 200-204.

RESUMO: O tromboembolismo venoso é uma causa comum de mortalidade e morbidade nos pacientes com câncer. Estes apresentam risco de 5-6 vezes maior de eventos tromboembólicos, comparados com a população geral. A cirurgia colorretal nesses pacientes implica em alto risco de complicações tromboembólicas pós-operatórias. Este artigo relata o caso de um paciente de 46 anos com câncer colorretal e carcinomatose peritoneal evoluindo com complicações tromboembólicas, além de revisão de literatura.

Palavras-chave: tromboembolismo venoso; câncer colorretal; cirurgia de intestino; tromboflebite.

\section{INTRODUÇÃO}

O tromboembolismo venoso (TEV) representa uma condição que se manifesta como trombose venosa profunda (TVP) e sua complicação, potencialmente fatal, é a embolia pulmonar (EP). É a principal causa de morte, com cerca de 1,5 a 2 milhões de casos anuais ${ }^{1}$.

O risco de TEV é significativamente aumentado nos pacientes com neoplasias malignas, sendo a incidência anual de 1:200 (vs. 1:1000 na população geral $)^{1}$. A possibilidade de complicações tromboembólicas aumenta, ainda mais, nos pacientes submeti- dos a operações abdômino-pélvicas, de forma especial na cirurgia colorretal.

O TEV acarreta impacto adverso significativo nos pacientes com câncer, incluindo diminuição da expectativa de vida, quando comparada à população geral. Assim, profilaxia e tratamento adequados são fundamentais.

A seguir relatamos o caso de um paciente com câncer colorretal e carcinomatose peritoneal evoluindo com complicações tromboembólicas, além de revisão de literatura.

\section{RELATO DE CASO}

Trabalho realizado no Hospital Governador Israel Pinheiro - Instituto de Previdência dos Servidores do Estado de Minas Gerais (IPSEMG) - Belo Horizonte (MG), Brasil.

Fonte de financiamento: não há

Conflito de interesse: nada a declarar

Recebido em: 20/05/2009

Aprovado em: 18/06/2009 
Trata-se de paciente do sexo masculino, 46 anos, casado, técnico em eletrônica, residente em área urbana, natural de Minas Gerais. Apresentou-se ao Serviço Médico de Urgência do Hospital Governador Israel Pinheiro em Belo Horizonte com relato de, há 1 mês, ter iniciado com dor abdominal e quadro de anemia importante. Referia hábito intestinal regular, com duas evacuações ao dia, fezes formadas, sem muco ou sangue. Apresentava sintomas secundários à anemia: palidez cutâneo-mucosa, dispneia aos esforços maiores e astenia. Negava uso diário de qualquer medicação, apenas analgésicos nos últimos dias. Alergia medicamentosa a sulfa. Na história pregressa, havia relato de quadro de hipertireoidismo quatro anos antes, controlado clinicamente, sem uso de medicamentos no momento. Nenhuma cirurgia prévia. Há quinze anos tinha estado internado para esclarecimento de dor abdominal, sem identificação de etiologia na ocasião.

Foi, então, internado por 10 dias para esclarecimento diagnóstico. À colonoscopia identificou-se lesão obstrutiva e endoscopicamente neoplásica em cólon direito. $\mathrm{O}$ anatomopatológico diagnosticou adenocarcinoma e o estadiamento não revelou metástases em outros órgãos. A seguir, foi encaminhado para tratamento cirúrgico. Submetido à laparotomia, ao inventário da cavidade foram identificados nódulos peritoneais pélvicos sugestivos de carcinomatose, além da lesão em cólon direito, sem evidências de disseminação neoplásica em outros sítios. Foram realizadas a colectomia direita com a anastomose primária íleocólica transversa e biópsias de uma das lesões peritoneais. Optou-se pela não abordagem destas lesões no mesmo tempo cirúrgico, em função das condições clínicas precárias do paciente (desnutrição e múltiplas transfusões). O exame anatomopatológico da peça cirúrgica confirmou adenocarcinoma colônico. O paciente evoluiu com íleo paralítico mais prolongado, ascite discreta, porém sem complicações maiores e recebeu alta hospitalar no $10^{\circ}$ dia pós-operatório (DPO).

Durante a preparação para reabordagem cirúrgica - peritoniectomia, três meses pós-operatório, em nível ambulatorial - foi realizada tomografia com emissão de positrons (PET-TC) para reestadiamento da doença. Neste exame identificou-se EP significativa (Figura 1), com vários trombos nas artérias pulmonares e, no entanto, o paciente negava dispneia, dor torácica ou quaisquer sintomas relacionados ao evento trom-

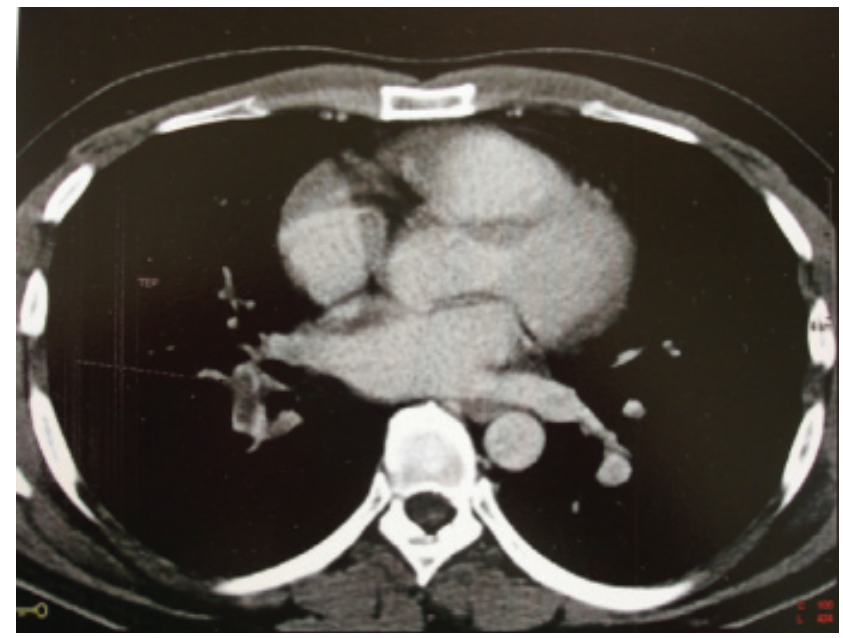

Figura 1. Tomografia computadorizada de tórax evidenciando embolia pulmonar (seta).

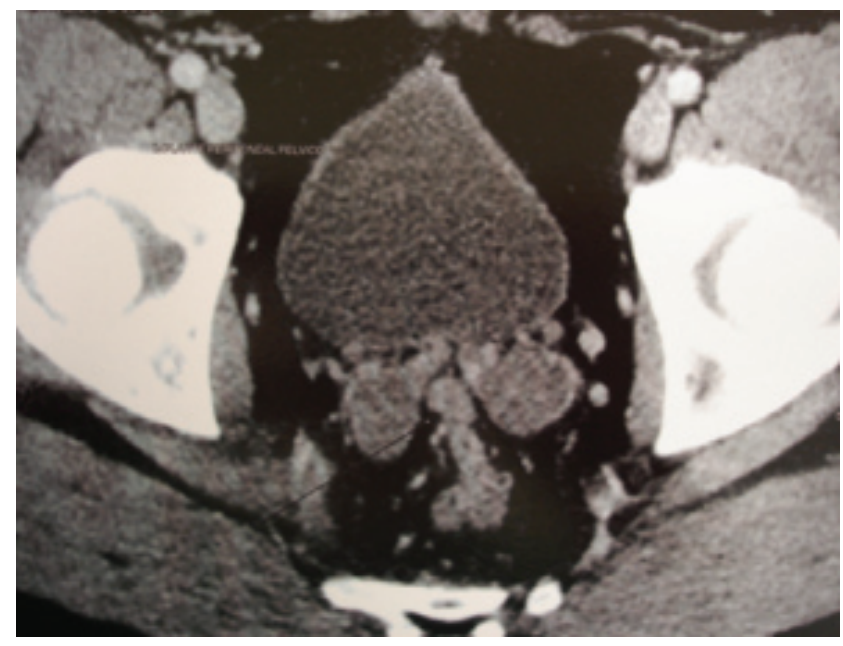

Figura 2. Tomografia computadorizada de pelve com implantes peritoneais (seta).

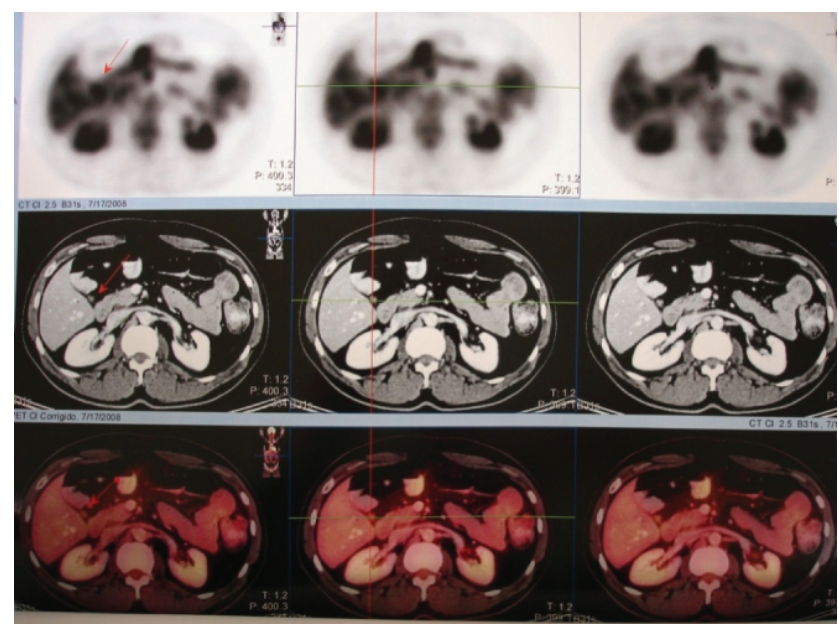

Figura 3. Tomografia com emissão de positrons com implante peritoneal em hilo hepático. 
boembólico. Ainda no PET-TC foi evidenciado, além dos nódulos pélvicos (Figura 2), vistos à cirurgia, implante em hilo hepático (Figura 3).

Diante do diagnóstico, o paciente foi anticoagulado e manteve Razão Normalizada Internaciona (RNI) entre 2,0 e 3,0, com uso regular de warfarin. Apesar disto, o paciente desenvolveu quadro de trombose venosa profunda sintomática em membro inferior direito, confirmada com Doppler (enquanto realizava exames cardiológicos para a cirurgia). Isto motivou seu encami-

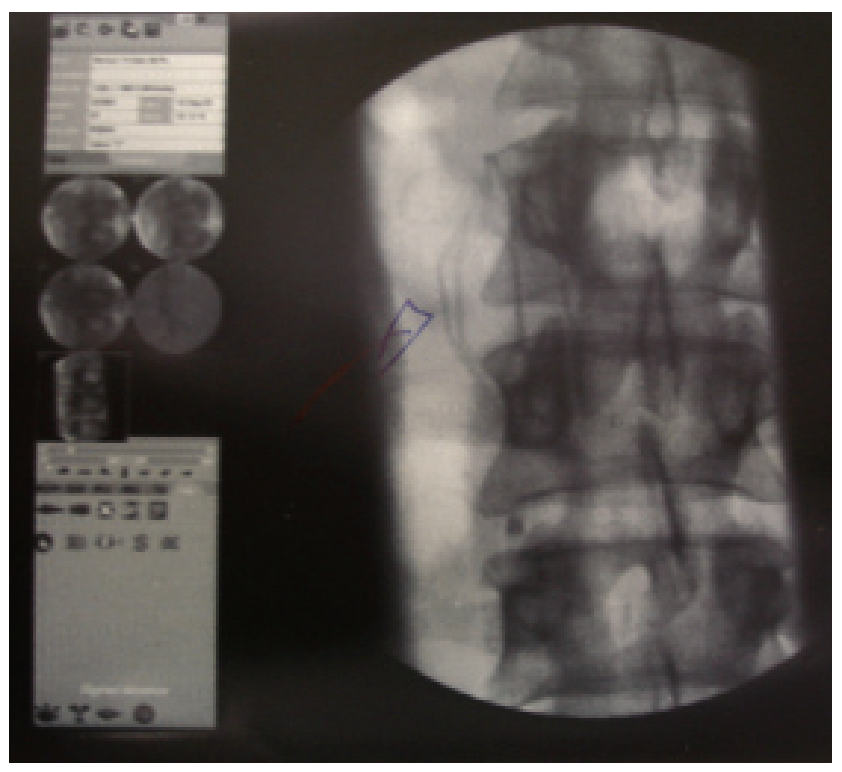

Figura 4. Implantação de filtro em veia cava inferior. nhamento para a equipe de cirurgia vascular que realizou implante de filtro em veia cava inferior (Figura 4).

Enfim, oito meses após a primeira cirurgia, foi realizada nova laparotomia para peritoniectomia. Durante este período, o paciente permaneceu em quimioterapia. Ao inventário da cavidade abdominal, foram identificados múltiplos implantes peritoneais em pelve, hilo hepático, goteira parietocólica direita, ureter direito, mesentério e alças de intestino delgado. Tais achados inviabilizaram o procedimento proposto inicialmente, sendo encerrada a operação sem abordagem dos implantes peritoneais.

Atualmente o paciente segue em uso regular de quimioterapia, mantendo estado geral preservado, após 14 meses desde a primeira intervenção cirúrgica.

\section{DISCUSSÃO}

Tradicionalmente, TVP e EP são consideradas doenças separadas; entretanto, evidências crescentes apontam que as duas doenças representam na verdade uma entidade clínica única. Entre os pacientes com TVP sintomática, 50-80\% apresentam evidência de EP assintomática. De forma semelhante, entre aqueles com EP sintomática pode ser demonstrado TVP assintomática em aproximadamente $80 \%$ das vezes ${ }^{2}$.

O TEV é uma causa comum de mortalidade e morbidade nos pacientes com câncer. Estes apresentam risco de 5-6 vezes maior de eventos tromboembólicos, comparados com a população geral. Os fatores de risco para TEV nos pacientes com neoplasia

Tabela 1. Fatores de risco de TEV nos pacientes com câncer.

\begin{tabular}{ll}
\hline Relacionados ao paciente & Idade acima de 40 anos \\
& História de TEV prévio \\
& Trombofilia \\
& Raça (maior nos afroamericanos) \\
Relacionados ao câncer & Período inicial após diagnóstico da neoplasia \\
& Sítio primário do câncer (TGI, cérebro, pulmão, pâncreas, renal, \\
& hematológico) \\
& Doença metastática \\
Relacionados ao tratamento & Operação recente \\
& Quimioterapia \\
& Terapia hormonal \\
& Uso de agentes antiangiogênicos \\
& Uso de agentes estimuladores da eritropoiese \\
& Hospitalização ou imobilização \\
& Cateter venoso central \\
&
\end{tabular}


maligna dependem das características do paciente, do câncer e do tipo de tratamento utilizado (Tabela 1). A quimioterapia representa um fator de risco independente de TEV. Está associada com incidência de até $2,2 \% /$ mês de quimioterapia. Nos casos de doença metastática, os riscos são ainda maiores. Esses pacientes apresentam até 20 vezes mais TEV que aqueles com doença neoplásica localizada e até 50 vezes mais do que pacientes sem câncer.

Estimativas sugerem que, na ausência de tromboprofilaxia, a incidência de TVP entre os pacientes submetidos a operações abdominais variam entre 15 $40 \%{ }^{4}$. A cirurgia colorretal implica em um risco ainda maior de complicações tromboembólicas, em comparação com a cirurgia geral. $\mathrm{O}$ tempo de duração prolongado, com dissecção pélvica e posicionamento per-operatório específico são fatores que explicam os riscos aumentados da cirurgia colorretal. Além disso, as indicações dessas operações, geralmente, são câncer ou doença inflamatória intestinal, ambas fatores de risco independentes de TEV. O câncer colorretal também está associado a uma frequência aumentada de trombofilia e a infecção de sítio cirúrgico, complicação comum em coloproctologia, é um importante fator de risco para TVP.

A tromboprofilaxia em pacientes com câncer deve ser sempre considerada, quando outros fatores de risco estão presentes, em especial imobilização e operação, podendo ser apropriada para todos pacientes com doença metastática.

Em relação à profilaxia farmacológica, heparina não-fracionada (HNF) e heparina de baixo peso molecular (HBPM) mostraram ser igualmente eficazes na prevenção de TVP e EP, como Borly et al. ${ }^{5}$ observaram em revisão sistemática da tromboprofilaxia na cirurgia colorretal no período entre 1970-2003. A profilaxia deve ser iniciada no pré-operatório e reintroduzida 12 a 24 horas após a operação. A profilaxia mecânica pode ser feita com meias de compressão graduada e compressão pneumática intermitente. É recomendada como adjuvante à profilaxia farmacológica nos pacientes de alto risco.

Evidências sugerem que eventos tromboembólicos tardios podem ocorrer até 7 semanas após a operação, e que cerca de $25 \%$ dos casos de EP ocorrem após a alta hospitalar. O estudo ENOXACAN II investigou a extensão da profilaxia com enoxaparina $40 \mathrm{mg} /$ dia por 1 semana e 4 semanas no pós-operatório de cirurgia oncológica abdominal e pélvica. A administração de enoxaparina por quatro semanas reduziu significativamente a incidência de TEV na quarta semana após a operação confirmada por venografia (4,8\% vs. $12 \%)$. Segundo o National Comprehensive Cancer Network (NCCN), o uso deve ser mantido por quatro semanas, na dose de $40 \mathrm{mg}$ ao dia, no pós-operatório ${ }^{6}$.

Apesar da reconhecida importância, a tromboprofilaxia é sistematicamente subutilizada nos hospitais, como exposto em estudo retrospectivo americano em pacientes de risco para TEV. Esse revelou taxas de profilaxia de apenas $25 \%$, mesmo nos pacientes oncológicos ${ }^{6}$. Essa profilaxia inadequada tem consequências graves e tem sido revista.

Já nos casos de TVP ou de EP, o tratamento inicial preferido é a HBPM, em relação à HNF. A preferência ocorre em função da eficácia similar, maior segurança sem necessidade de monitorização laboratorial, menor mortalidade particularmente nos pacientes com câncer, menor risco de trombocitopenia induzida pela heparina e comodidade posológica com uso uma ou duas vezes ao dia, por três a seis meses.

Pacientes com câncer e TEV apresentam maior chance de recorrência, tanto em uso de anticoagulantes, como após completado o tratamento. $\mathrm{O}$ uso terapêutico prolongado (3-6 meses) de HBPM em oposição ao manejo tradicional com warfarin, nesse grupo de pacientes, está associado com uma redução importante da recorrência tromboembólica $(9 \% \text { vs } 17 \%)^{3}$. $\mathrm{O}$ tratamento continua enquanto o câncer permanecer ativo, isto é, até a cura.

O filtro de veia cava, preferencialmente removível, é indicado para pacientes com contraindicações ao uso de anticoagulante e naqueles com episódio de TEV em uso de anticoagulante.

Assim, ao tratar pacientes oncológicos colorretais, o médico deve estar atento para realizar adequada profilaxia para TEV e para diagnosticar provável EP. A manutenção da profilaxia não deve ser negligenciada embora o custo financeiro disto possa ser um empecilho, quando se considera o uso de HBPM. Porém, os riscos da TVP/EP são reais e justificam os cuidados na sua prevenção, desafio para o cirurgião. 
ABSTRACT: Venous thromboembolic disease is a common cause of mortality and morbidity in patients with cancer. Patients have a 5-6 fold increase in the risk for a venous thromboembolism compared with the general population. Colorectal surgery in these patients implies a specific high risk for postoperative thromboembolic complications. This article reports a case of a 46 years old patient with colorectal cancer and peritoneal carcinomatosis developing thromboembolic complications, and literature review.

Keywords: venous thromboembolism; colorectal cancer; intestine surgery; thrombophlebitis.

\section{REFERÊNCIAS}

1. Jacobson A. Venous Thromboembolic disease in cancer patients: a satellite symposium summary. Dialogues in Clinical Practice 2008;1-12.

2. Buller HR, Sohne M, Middeldorp S. Treatment of venous thromboembolism. J Thromb Haemost 2005;3(8): 1554-60.

3. Fennerly A. Venous thromboembolic disease and cancer. Postgrad Med J 2006;82(972):642-8.

4. Bergqvist D. Venous Thromboembolism: a review of risk and prevention in colorectal surgery patients. Dis Colon Rectum 2006;49(10):1620-8.
5. Borly L, Wille-Jorgensen P, Rasnussen MS. Systematic review of thromboprophylaxis in colorectal surgery - an update. Colorectal Dis 2004;7(2):122-7.

6. Khorana AA. The NCCN clinical practice guidelines on venous thromboembolic disease: strategies for improving VTE prophylaxis in hospitalized cancer patients. Oncologist 2007;12(11):1361-70.

Endereço para correspondência:

Sinara Mônica de Oliveira Leite

Av. Contorno 4.747/912 - Condomínio Lifecenter - Serra

CEP: 30110090 - Belo Horizonte (MG), Brasil

E-mail: sinara.monica@uol.com.br 